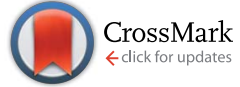

Cite this: RSC Adv., 2017, 7, 7725
Received 29th November 2016 Accepted 9th January 2017

DOI: $10.1039 / c 6 r a 27475 g$

www.rsc.org/advances

\section{Inkjet printed polystyrene sulfuric acid-doped poly(3,4-ethylenedioxythiophene) (PEDOT) uniform thickness films in confined grooves through decreasing the surface tension of PEDOT inks}

\author{
Rubo Xing, Shumeng Wang, Baohua Zhang, Xinhong Yu, Junqiao Ding, Lixiang Wang \\ and Yanchun Han*
}

A uniform thickness of inkjet printed polystyrene sulfuric acid-doped poly(3,4-ethylenedioxythiophene) (PEDOT) films in confined grooves is very important for their application in inkjet printed polymer lightemitting displays (PLEDs). PEDOT films with a concave or convex cross section are often obtained during inkjet printing. We obtained uniform thickness printed PEDOT films through the low surface tension modification of PEDOT inks using isopropanol (IPA). The thickness fluctuation along the cross section of the PEDOT films was decreased from $\sim 30 \mathrm{~nm}$ for the convex condition and $\sim 20 \mathrm{~nm}$ for the concave condition to less than $10 \mathrm{~nm}$ through replacing the original PEDOT ink with 10\% IPA modified PEDOT ink. The meniscus profile at gelation of the PEDOT inks was used to explain the morphology change of the PEDOT films. IPA modification of PEDOT inks induced lower surface tension and increased the Bond number, which decreased the curvature of the meniscus and improved film uniformity both under concave and convex conditions. IPA modification has no obvious effect on the PEDOT morphology, work function and performance of organic light-emitting diodes.

\section{Introduction}

Organic/polymer light-emitting diodes have exhibited tremendous potential for use in flat panel displays as a result of their fast response time, high contrast, and compatibility with low cost printing processes and light-weight flexible substrates. The inkjet printing approach for manufacturing organic/polymer light-emitting displays (O/PLEDs) has attracted great attention in the last few years because of the non-contact digital printing process, suitability for large area flexible substrates, low cost at high speed, and so on. ${ }^{1-5}$ The morphological properties of printed photoelectric films, such as uniformity of thickness and patterning resolution, are of great importance in fabricating reliable printed $\mathrm{O} / \mathrm{PLEDs}$ and improving their performance.

The solute prefers to deposit at the rim for inkjet printed dots. A ring-like morphology can form after the drying process. ${ }^{6}$ This phenomenon is known as the coffee ring effect, which has been utilized to form electrodes for organic thin film transistors and transparent electrodes on flexible substrates. ${ }^{7-9}$ But the elimination of the coffee ring effect is necessary for inkjet

State Key Laboratory of Polymer Physics and Chemistry, Changchun Institute of Applied Chemistry, Chinese Academy of Sciences, 5625 Renmin Street, Changchun 130022, People's Republic of China. E-mail: ychan@ciac.ac.cn; Fax: +86-43185262126; Tel: +86-431-85262175 printing of O/PLEDs in order to form uniform films in the printed pixels, which are useful for obtaining an increased ratio of light emitting area in every pixel and electrical uniformity improvement in display panels.

Deegan and his coworkers ${ }^{\mathbf{1 0}, 11}$ have researched the coffee ring effect deeply and provide a convincing mechanism for this phenomenon. At first, the contact line of a droplet needs pinning on the surface during its drying process. Fastest evaporation occurs at the edge of the droplet, because of the higher degree of diffusion freedom of solvent vapor in the corresponding region. Then, capillary flow from the center to the edge forms, to supply solvent for evaporation loss, which transports solute to the edge of droplet, where it is deposited. The coffee-ring effect occurs. Therefore, control of flow behavior in drying droplets can be key in eliminating the coffee ring effect.

Numerous significant methods have been explored and used to eliminate the coffee ring effect. Marangoni flow ${ }^{\mathbf{1 2}}$ can be an effective way to eliminate the coffee ring effect. Marangoni flow emerges from a region of low surface tension to one of high surface tension when there is a surface tension gradient along the droplet surface. Formation of an inward Marangoni flow in the droplet can be used to avoid the coffee ring effect caused by outward capillary flow. As droplets of solvent mixture evaporate, a solvent component gradient can be formed along the radical direction of the droplet, which establishes a surface tension 
gradient and drives Marangoni flow. Solvent mixture induced inward Marangoni flow has been utilized to adjust the morphology of printed polymer dots and conductive silver nanoparticle lines. ${ }^{13-15} \mathrm{Hu}$ et al. ${ }^{12,16}$ also formed inward Marangoni flow through a temperature induced surface tension gradient in drying droplets and realized reversed coffee ring deposition.

Solutions with high viscosity or even ones that undergo gelation can provide another popular and effective way of eliminating the coffee ring effect. The working mechanism comes from decreased intensity of capillary flow as a result of the ink viscosity. Utilizing the sol-gel transition of inks, Schubert et al. ${ }^{17}$ and Bain et al. ${ }^{\mathbf{1 8}}$ realized uniform deposition of $\mathrm{TiO}_{2}$ nanoparticles and LAPONITE® respectively. Uniform polymer films have also been printed using inks containing a high viscosity and low volatility solvent component, combined with control of the drying velocity. ${ }^{19-22}$

Polystyrene sulfuric acid-doped poly(3,4-ethylenedioxythiophene) (PEDOT) is widely used as a hole injection layer in the fabrication of O/PLEDs. The main research into the inkjet printing of PEDOT films has focused on conductivity promotion through the modification of PEDOT inks to realize ITO free devices. ${ }^{23-28}$ Rare works have focused on morphology control of inkjet printed PEDOT films. ${ }^{29,30}$ Thickness and film uniformity of inkjet printed PEDOT films is very important in ensuring the quality of the next deposited organic light emitting films beyond PEDOT films and the performance of printed O/PLEDs.

In this study, methods and mechanisms for the inkjet printing of uniform PEDOT films in confined grooves forming low surface energy banks have been researched. Inkjet printing assisted by low surface energy banks has been used as an effective and necessary way of confining free wetting and spreading of inks droplets and improving patterning resolution in the inkjet printing of O/PLEDs. But the thickness uniformity of the printed films would be affected dramatically by low surface energy banks because of meniscus configuration compared with a normal flat substrate. Uniform PEDOT films were printed in a wider range of thicknesses, through low surface tension modification of PEDOT inks, and were used for the fabrication of OLEDs.

\section{Experimental section}

\subsection{Materials}

The polystyrene sulfuric acid-doped poly(3,4-ethylenedioxythiophene) (PEDOT, CLEVIOS ${ }^{\mathrm{TM}}$ P VP AI4083) was purchased from Heraeus. Isopropanol (IPA) was purchased from Acros and used without further purification. ITO glass (glass slide with indium-tin-oxide film) was purchased from Chinese South Glass Holding (CSC) Co., Ltd. The negative photoresists were purchased from Futurrex. The organic light emitting materials, including dendritic host $\mathrm{H} 2$, green phosphor $\operatorname{Ir}(\mathrm{PBI})_{3}$, and electron transporting SPPO13, were prepared according to literature methods. ${ }^{31-33}$

\subsection{Preparation of the substrate}

Photoresists were spin coated on cleaned ITO glass. The thickness of the photoresist films was adjusted through altering the spin coating speed combined with the concentration of the photoresist. Photoresist banks of different heights can be formed on the ITO glass substrate after a UV-exposure and developing process. The width of the banks is $50 \mu \mathrm{m}$ and the width of the grooves between banks, which consist of the bare ITO surface, is $200 \mu \mathrm{m}$. A substrate with hydrophilic ITO grooves (contact angle of water: $<20^{\circ}$ ) and hydrophobic photoresist banks (contact angle of water: $>100^{\circ}$ ) can be formed after modification using oxygen plasma and CFx plasma continuously.

\subsection{Inkjet printing of PEDOT films and OLED fabrication}

An inkjet printer (Microdrop Technique GmbH, AD-P-8000 system) was used for the printing of PEDOT films and organic light-emitting films. The inkjet print heads have an inner diameter of about $70 \mu \mathrm{m}$. PEDOT solution was supplied to the print head at first. Stable droplets with a diameter of about 80 $\mu \mathrm{m}$ can be formed at a jetting frequency of $200 \mathrm{~Hz}$ through the adjustment of the waveform. The PEDOT droplets were printed into the hydrophilic ITO grooves between the hydrophobic photoresist banks. The distance of the droplets was used to adjust the volume of deposited PEDOT inks and the corresponding film thickness. The printed PEDOT inks were dried under a standard atmosphere and at room temperature to form PEDOT films at first. Then, the PEDOT films were baked at $130{ }^{\circ} \mathrm{C}$ for 1 hour for deep drying and used for profile measurement and the fabrication of devices.

Another organic light emitting layer was inkjet printed on the PEDOT films for the fabrication of OLEDs. The electron transport layer (SPPO13) and $\mathrm{LiF} / \mathrm{Al}$ cathode were deposited on the organic light emitting films using physical vapor deposition.

\subsection{Characterization}

The surface tension of the PEDOT inks was measured with a contact angle measuring system (DSA-10, Kruss) used its pendant drop mode. The inkjet printed PEDOT films were observed using an optical microscope (Leica DMLP, Leica Microsystems Ltd., Germany). The thickness profiles of the PEDOT films were investigated using a Veeco Dektak 6M Stylus Profilometer. The surface morphology of the PEDOT films was observed using an atomic force microscope (SPA 300HV with an SPI 3800N Probe Station, Seiko Instruments Inc., Japan) in tapping mode. Ultraviolet photoemission spectroscopy (UPS) was carried out using a helium discharge lamp ( HeI $\alpha=21.2 \mathrm{eV}$ ) and a hemispherical energy analyzer (Specs PHOIBOS 150) to test the work function of the PEDOT films. The brightnessvoltage characteristics of the OLEDs were recorded using a computer-controlled sourcemeter (Keithley 2400) at room temperature under ambient conditions.

\section{Results and discussion}

\subsection{Printing of uniform PEDOT films}

The low surface energy banks, which have a height of $1.9 \mu \mathrm{m}$, were used to assist the inkjet printing of PEDOT films at first. PEDOT inks at different volumes, including $6 \mathrm{pL} \mathrm{mm}^{-2}, 10 \mathrm{pL}$ $\mathrm{mm}^{-2}$ and $16 \mathrm{pL} \mathrm{mm} \mathrm{m}^{-2}$, were deposited into the grooves 

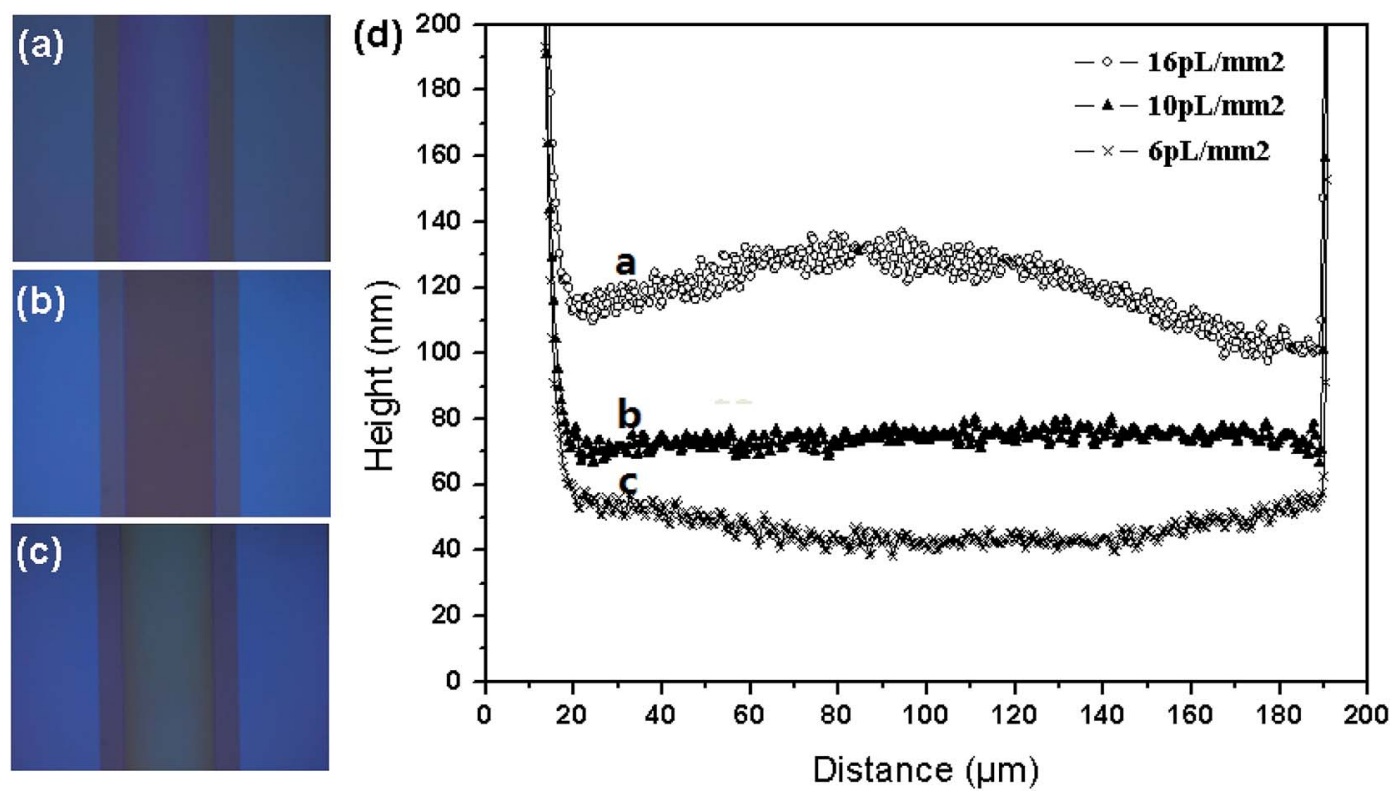

Fig. 1 Optical images of PEDOT films deposited with different ink volumes: (a) $16 \mathrm{pL} \mathrm{mm}^{-2}$, (b) $10 \mathrm{pL} \mathrm{mm} \mathrm{m}^{-2}$, and (c) $6 \mathrm{pL} \mathrm{mm}{ }^{-2}$; and (d) their cross-sectional profiles in grooves with $1.9 \mu \mathrm{m}$ banks.

between the low surface energy banks. PEDOT inks filled the grooves and were confined in them. Overflow did not happen at all under these conditions. PEDOT films were obtained in the grooves after the drying process. Fig. 1 shows optical images of the dried PEDOT films and their cross sectional profiles. PEDOT film with a convex cross section was obtained when a PEDOT ink volume of about $16 \mathrm{pL} \mathrm{mm}^{-2}$ was deposited, as shown in Fig. 1(d). The gradual color change of the PEDOT film, as shown in Fig. 1(a), also demonstrates the gradual change in thickness and is in accordance with the results of the cross section profile. On the other hand, uniform PEDOT film was obtained when a PEDOT ink volume of about $10 \mathrm{pL} \mathrm{mm}^{-2}$ was deposited. The corresponding PEDOT film also displays a uniform color, as shown in Fig. 1(b). PEDOT film with a concave cross-section was

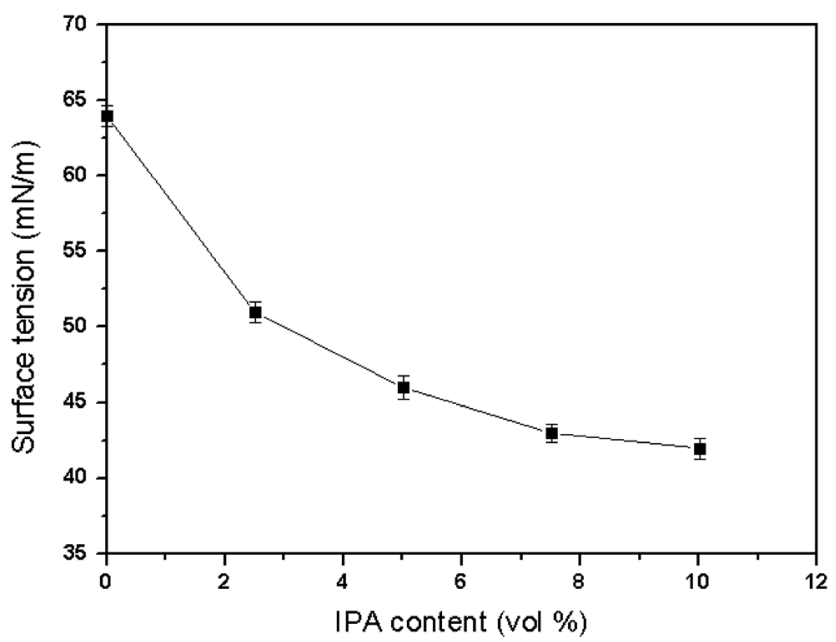

Fig. 2 Relationship between IPA content in PEDOT inks and their surface tension. obtained when a PEDOT ink volume of about $6 \mathrm{pL} \mathrm{mm}^{-2}$ was deposited.

Alcohol has been used to modify PEDOT and increase its electrical conductivity in previous research. ${ }^{34}$ In this research, isopropanol (IPA) was added to the PEDOT ink to improve the uniformity of the inkjet printed PEDOT films, due to its excellent properties for decreasing ink surface tension and ensuring the jetting stability of the PEDOT solution. PEDOT inks modified with less than 10\% IPA have stable solubility and jetting properties. The surface tension of PEDOT inks with different IPA content has been investigated. Fig. 2 shows the relationship curve between IPA content in PEDOT inks and their surface tension. The surface tension of pure IPA is about $21.7 \mathrm{mN} \mathrm{m}^{-1}$. The surface tension of the original PEDOT inks is about 64.0 $\mathrm{mN} \mathrm{m}{ }^{-1}$. There is an obvious decrease in the surface tension of IPA modified PEDOT inks with an increase in IPA content because of the low surface tension of IPA.

PEDOT inks with different IPA content, including 0\%, 2.5\%, $5.0 \%, 7.5 \%$ and $10.0 \%$, were printed into grooves with $1.9 \mu \mathrm{m}$ banks to test the film uniformity. Fig. 3 shows optical images of dried PEDOT films deposited at an ink volume of $6 \mathrm{pL} \mathrm{mm}^{-2}$ and their cross sectional profiles. The uniformity of the printed PEDOT improved gradually with increasing the IPA content of the PEDOT inks, as shown in Fig. 3(f). The thickness fluctuation along the cross section of the PEDOT films decreased from $\sim 20 \mathrm{~nm}$ to less than $10 \mathrm{~nm}$ through replacing the original PEDOT ink with $10 \%$ IPA modified PEDOT ink. The results from the optical images, as shown in Fig. 3(a)-(e), are in accordance with data from the cross-section profile.

Similar phenomena have also been observed for PEDOT films deposited at $16 \mathrm{pL} \mathrm{mm} \mathrm{mm}^{-2}$. Fig. 4 shows optical images of dried PEDOT films and their corresponding cross-sectional profiles. The thickness uniformity of printed PEDOT has been 

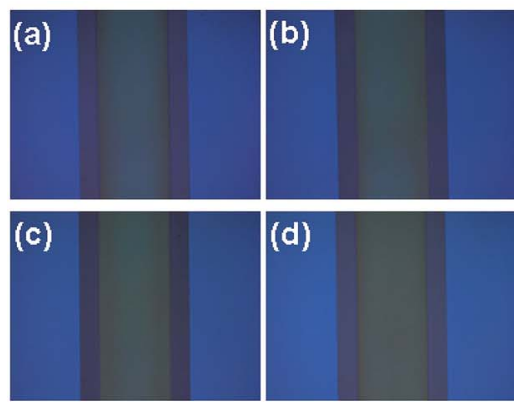

(d)

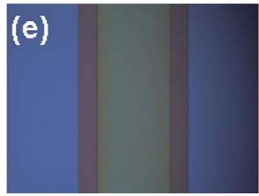

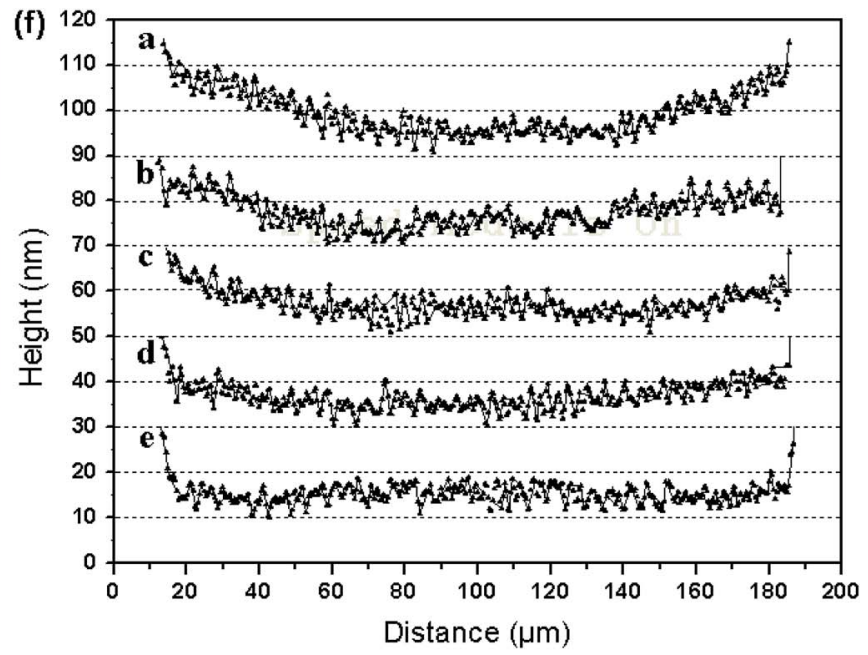

Fig. 3 Optical images of PEDOT films deposited at an ink volume of $6 \mathrm{pL} \mathrm{mm} \mathrm{m}^{-2}$ from PEDOT inks with different IPA content: (a) $0 \%$, (b) $2.5 \%$, (c) $5.0 \%$, (d) $7.5 \%$, and (e) $10.0 \%$; and (f) their corresponding cross-sectional profiles in grooves with $1.9 \mu \mathrm{m}$ banks.
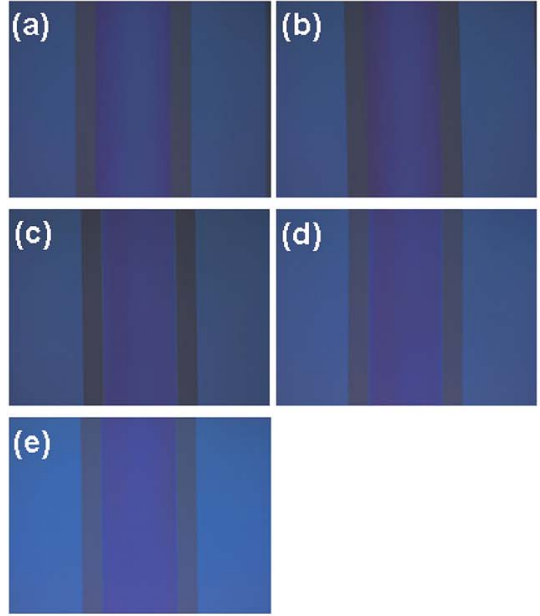

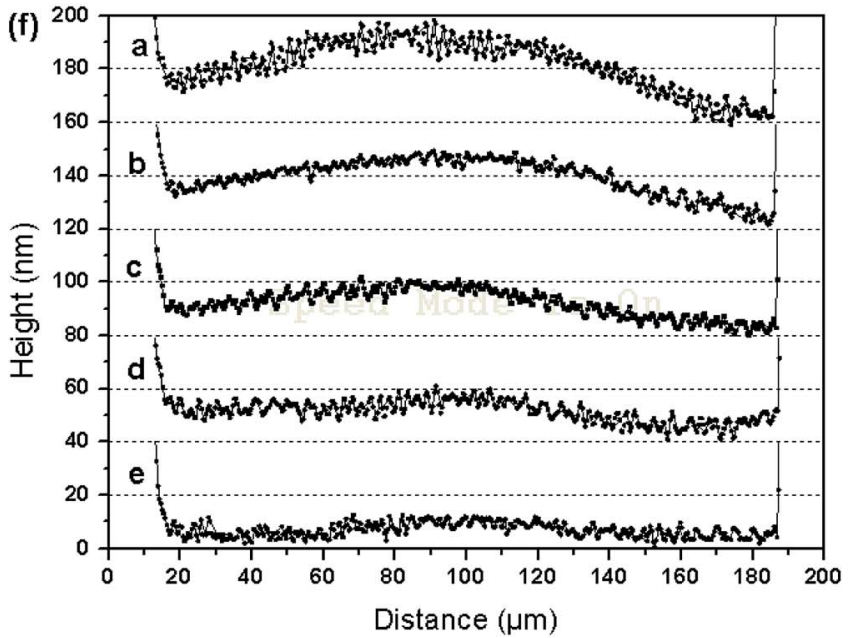

Fig. 4 Optical images of PEDOT films deposited at an ink volume of $16 \mathrm{pL} \mathrm{mm} \mathrm{mm}^{-2}$ from PEDOT inks with different IPA content: (a) $0 \%$, (b) $2.5 \%$, (c) $5.0 \%$, (d) $7.5 \%$, and (e) $10.0 \%$; and (f) their corresponding cross-sectional profiles in grooves with $1.9 \mu \mathrm{m}$ banks.

improved gradually with increasing the IPA content of PEDOT inks. The thickness fluctuation along the cross section of the PEDOT films has decreased from $\sim 30 \mathrm{~nm}$ to less than $10 \mathrm{~nm}$ through replacing the original PEDOT inks with 10\% IPA modified PEDOT inks. The results from the optical images, as shown in Fig. 4(a)-(e), are in accordance with the cross-section profiles. It is clear that IPA modified PEDOT inks have improved film uniformity. Modified PEDOT inks with 10\% IPA content have shown the most excellent properties for improving the thickness uniformity.

Low surface energy banks with a height of $3.5 \mu \mathrm{m}$ were also used to assist inkjet printing. Original and 10\% IPA modified PEDOT ink was deposited into the grooves, respectively, to investigate the film profiles. Fig. 5 shows optical images of dried PEDOT films and their corresponding cross-sectional profiles. Uniform PEDOT films were obtained when PEDOT ink at a volume of about $30 \mathrm{pL} \mathrm{mm} \mathrm{mm}^{-2}$ was deposited. PEDOT films with convex and concave cross sections were also formed at higher $\left(38 \mathrm{pL} \mathrm{mm} \mathrm{m}^{-2}\right)$ and lower $\left(22 \mathrm{pL} \mathrm{mm}^{-2}\right)$ deposition amounts of PEDOT inks. But it is obvious that PEDOT films deposited using 10\% IPA modified PEDOT ink show lower thickness fluctuation and improved thickness uniformity. This regularity is similar to the results obtained from printing on a substrate with $1.9 \mu \mathrm{m}$ banks.

\subsection{Mechanism for the improvement of film uniformity}

Low surface energy banks affect the thickness uniformity of inkjet printed PEDOT films dramatically, from the results above. The amount of deposited PEDOT ink needs to be matched with the bank to realize the printing of uniform PEDOT films. There is an optimal volume of deposited PEDOT ink for a certain bank to obtain uniform PEDOT films. Uniform PEDOT films can be obtained only over a narrow range of 

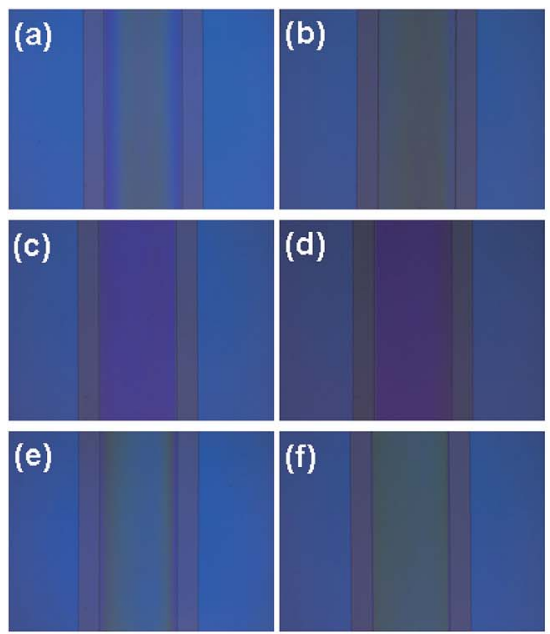

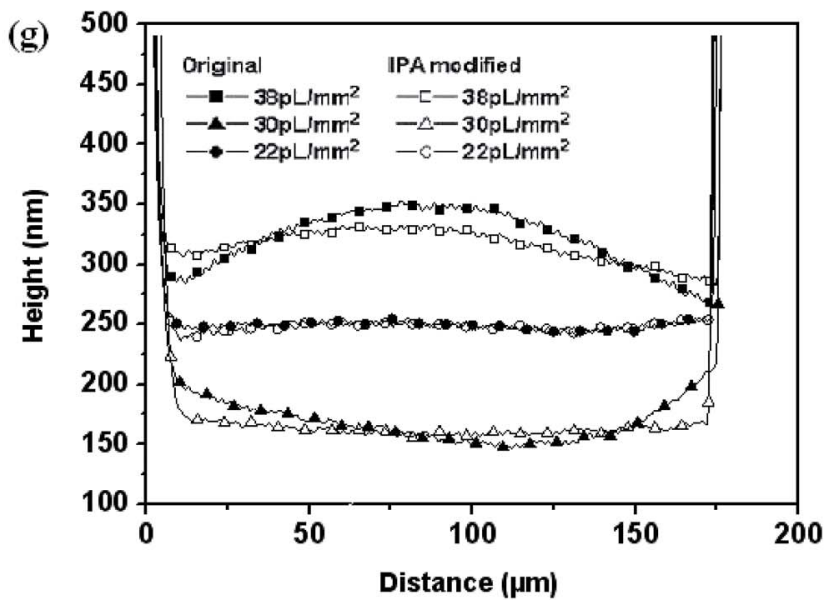

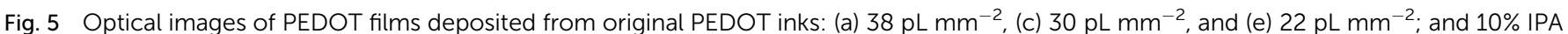
modified PEDOT inks: (b) $38 \mathrm{pL} \mathrm{mm}{ }^{-2}$, (d) $30 \mathrm{pL} \mathrm{mm}^{-2}$, and (f) $22 \mathrm{pL} \mathrm{mm}^{-2}$; and (g) their corresponding cross-sectional profiles in grooves with $3.5 \mu \mathrm{m}$ banks.

thicknesses for a given bank. PEDOT films with a concave (or convex) cross-section would be obtained if PEDOT ink volumes less (or more) than the optimal amount were deposited. Therefore, the volume of deposited PEDOT ink needs to be tested and controlled accurately for a certain bank to insure the uniformity of PEDOT films. Otherwise, PEDOT films with large thickness fluctuations would emerge.

More uniform PEDOT films have been printed from PEDOT ink modified with IPA compared with original PEDOT ink under the same experimental conditions, from the results above. Uniform PEDOT films can be obtained at a wider range of thicknesses from IPA modified PEDOT ink for a given bank. This means that the range of matching between volumes of printed PEDOT ink and bank height increased, which is very important for practical application. The mechanism relating to this improvement will be discussed below.

From previous works, ${ }^{17,18}$ gelation can be an effective way to eliminate the coffee-ring effect in inkjet printed dots and films. This principle can be used to explain our experimental phenomena relating to matching between volumes of printed PEDOT ink with bank height. The viscosity of polymer solutions would increase dramatically with an increase in their concentration normally. The viscosity of PEDOT ink can be increased from $4 \mathrm{mPa}$ s to $20 \mathrm{mPa} \mathrm{s}$ when the concentration increases from $1.3 \%$ to $1.7 \%$, based on data from a supplier of PEDOT inks. It is obvious that a dramatic increase in viscosity would emerge with the evaporation of printed PEDOT ink. Gelation would happen when the viscosity is high enough. The flowability of PEDOT ink vanishes at gelation. Dried films would maintain the profile of the meniscus at gelation.

$$
B=\rho g r^{2} / \sigma
$$

The Bond number is defined, using eqn (1), as the most significant factor in estimating the profile of the meniscus, where $\rho$ is the density of the ink, $r$ is the half width of the grooves, $\sigma$ is the surface tension of the ink and $g$ is the acceleration due to gravity. Fig. 6 provides the Bond numbers of PEDOT inks with different IPA content in our experiments. All the inks have small Bond numbers. A meniscus with a spherical profile would form from normal experience for inks with such small Bond numbers.

A PEDOT gel with a convex meniscus would form if a large amount of PEDOT ink was deposited in the grooves, as shown in Fig. 7(a), where $G$ is gravity, and $\sigma$ is the surface tension of the ink. Then, PEDOT film with a convex cross section can be obtained in the grooves after the drying process. Certainly, PEDOT gel with a flat (Fig. 7(b)) and concave (Fig. 7(c)) meniscus can be obtained in the grooves with a decrease in the volume of printed PEDOT ink. Films with flat and concave cross sections can be obtained correspondingly. This explains the phenomenon relating to the morphology changes of PEDOT films with a decrease in the volume of printed PEDOT ink in the grooves, as shown in Fig. 1 and 5. It also clarifies that the key to printing flat PEDOT films in the low surface energy banks formed by confined grooves is to match the printed ink volume to the height of the bank.

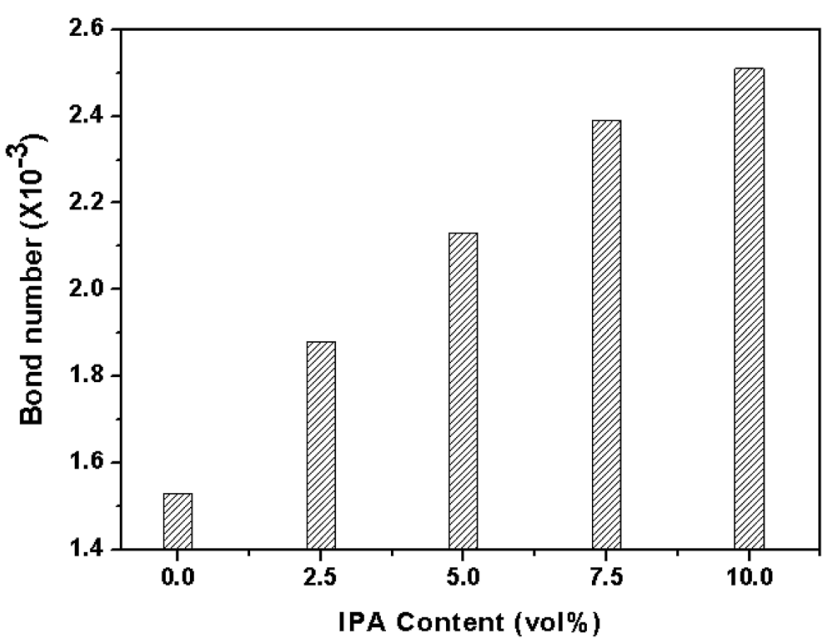

Fig. 6 Bond numbers of PEDOT inks with different IPA content. 


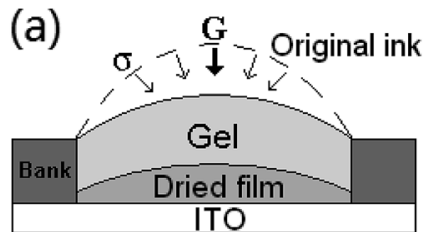

(b)

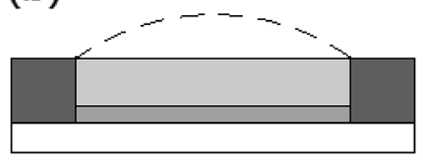

(c)

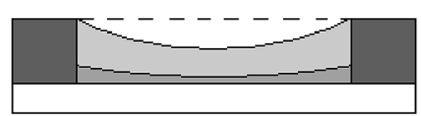

IPA modified

IPA

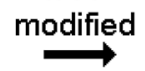

IPA modified (d)

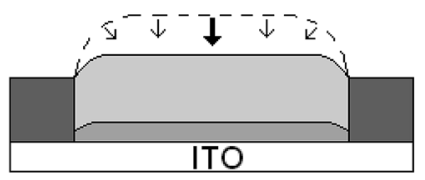

(e)

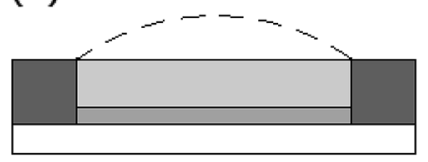

(f)

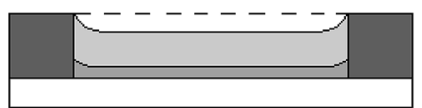

Fig. 7 Sketch map detailing profile changes in printed PEDOT films with a decrease in ink volume and surface tension. PEDOT films with (a) convex, (b) flat and (c) concave cross section can be obtained with decrease of volume of printed inks. PEDOT films with (d) flattened convex, (e) flat and (f) flattened concave cross section can be printed at the same condition expect the using of low surface energy modified inks.
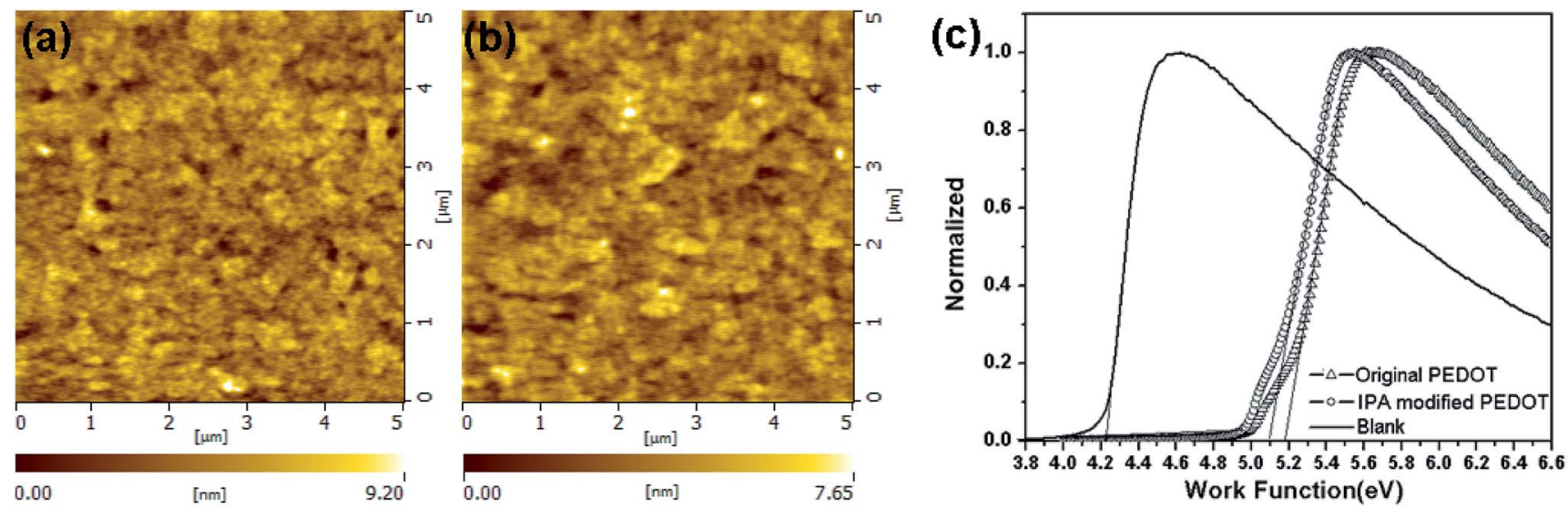

Fig. 8 AFM images of PEDOT films deposited from (a) original PEDOT ink and (b) 10\% IPA modified PEDOT ink and (c) their work functions.

There is an obvious increase in the Bond number with an increase in the IPA content in modified PEDOT inks because of a decrease in the surface tension. The ratio between gravity and the surface tension would increase, which induces a meniscus with a more flat top surface, as shown in Fig. $7(d)$ and (f). Uniformity of printed PEDOT films can be improved compared with films deposited from non-modified PEDOT inks. Certainly, there are no obvious differences for gels with a flat meniscus, as shown in Fig. 7(b) and (e). The experimental results, as shown in Fig. 5 for PEDOT films deposited from original and modified PEDOT inks at $30 \mathrm{pL} \mathrm{mm}^{-2}$, are also consistent with this opinion.

Under normal conditions, the meniscus is spherical for such small Bond numbers. But the thickness fluctuations in PEDOT films are also very small. There is less than $20 \mathrm{~nm}$ thickness difference across printed PEDOT films about $160 \mu \mathrm{m}$ wide. The meniscus curvature is very low correspondingly. This may be the main reason for obtaining uniform PEDOT films over a wide thickness range of banks from low surface energy modified PEDOT inks.

\subsection{Electronic performance of PEDOT films}

To demonstrate the effect of IPA modification on the performance of PEDOT films, we tested their morphology and work function first. Fig. 8(a) and (b) provide AFM images of PEDOT films deposited from original and 10\% IPA modified PEDOT ink. All the PEDOT films are smooth. The surface roughness of PEDOT film deposited from original and IPA modified PEDOT ink is about $1.29 \mathrm{~nm}$ and $1.14 \mathrm{~nm}$, respectively. There is no obvious difference between their surface morphologies. Fig. 8(c) provides the work functions of PEDOT films. There is a slight decrease in the HOMO level for PEDOT films deposited from IPA modified ink compared with those deposited from the original PEDOT ink. 
(a)

(c)
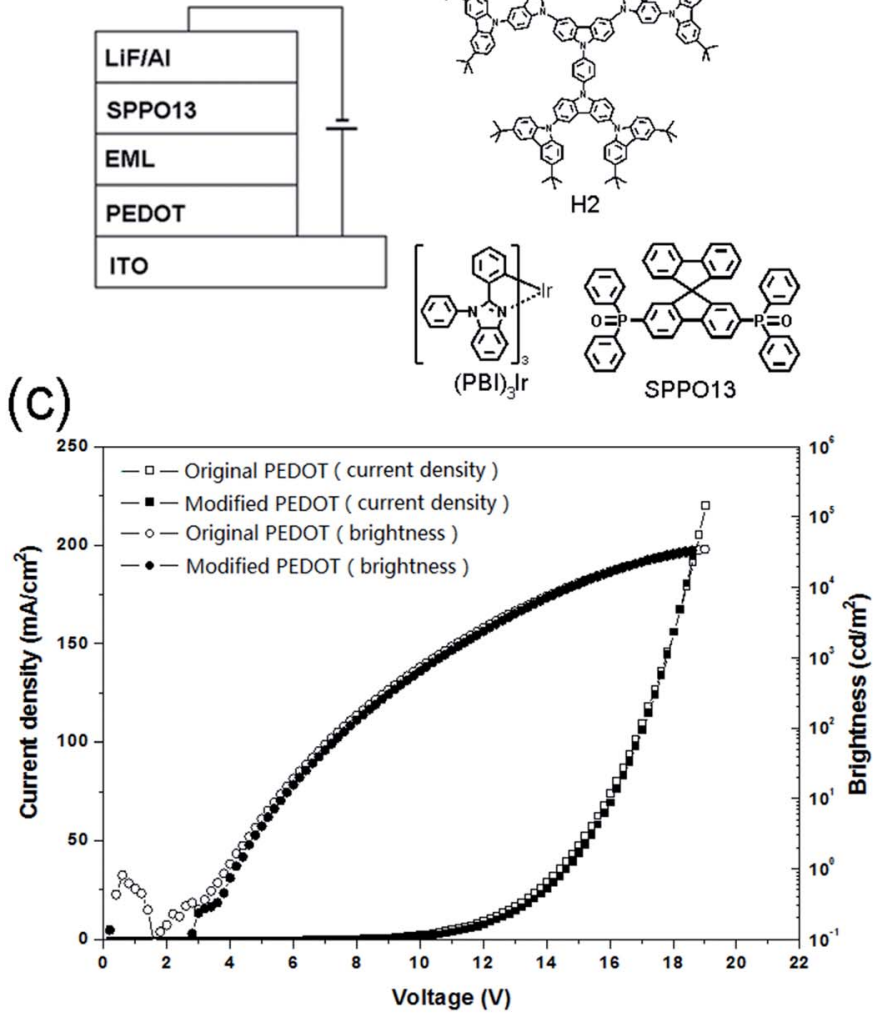

(b)

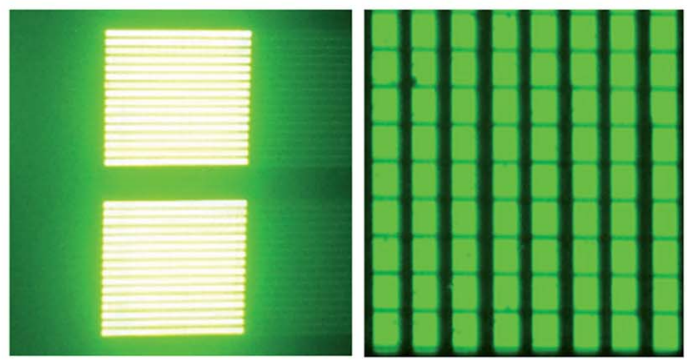

(d)

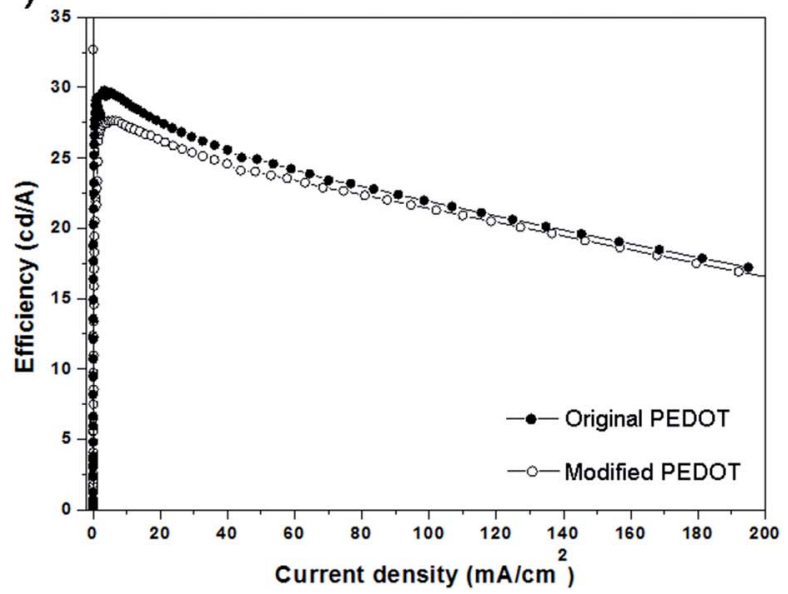

Fig. 9 (a) Configuration of devices and the chemical structure of the materials. (b) Optical image and detailed pixel matrix of the electroluminescent device under working conditions. (c) Voltage-current density-brightness relationship and (d) current density-efficiency relationship of the electroluminescent devices.

The PEDOT films were also used for fabrication of electroluminescent devices. Fig. 9(a) shows the device configuration and the chemical structure of the materials. The emissive layer (EML) is comprised of the green phosphorescent dye $\operatorname{Ir}(\mathrm{PBI})_{3}$ doped in a dendritic H2 host. Dendritic H2 is selected as the host because of its high triplet level $(2.89 \mathrm{eV})$ and satisfactorily high-lying highest occupied molecular orbital (HOMO) level. Fig. 9(b) shows an optical image of the electroluminescent device and the detailed pixel matrix in working condition, which used PEDOT films printed from 10\% IPA modified PEDOT inks as the hole injection layer. The luminous intensity of the pixels and matrix are all smooth, which proved the uniformity of the PEDOT films in pixel degree and matrix degree. Fig. 9(c) and (d) also provide the electronic properties of the electroluminescent devices. All the devices have similar performance. A small difference in the efficiency, as shown in Fig. 9(d), may come from experimental error. There is no obvious effect on the electronic performance of OLEDs through IPA modification of PEDOT inks.

\section{Conclusion}

In summary, we report a method to improve the thickness uniformity of inkjet printed and drying-confined PEDOT films through a simple low surface tension modification of PEDOT ink. The amount of deposited PEDOT ink must match with the bank height exactly to obtain uniform PEDOT films and to avoid films with concave or convex cross sections. Through low surface tension modification of PEDOT inks using IPA, the thickness fluctuations in PEDOT films have been decreased obviously both under concave and convex conditions. The thickness fluctuations along the cross section of the PEDOT films has decreased from $\sim 30 \mathrm{~nm}$ under convex conditions and $\sim 20 \mathrm{~nm}$ under concave conditions to less than $10 \mathrm{~nm}$, through replacing the original PEDOT ink with $10 \%$ IPA modified PEDOT ink. The meniscus profile at gelation of the PEDOT ink can be key to deciding uniformity of the PEDOT films confined by grooves. IPA modification of PEDOT ink induced low surface tension and increased the Bond number, which decreased the curvature of the meniscus and improved the film uniformity. The electronic properties of electroluminescent devices, using PEDOT films deposited from original and IPA modified inks as the hole injection layer, have similar performances. There is no obvious effect on the electronic performance of PEDOT films as a result of IPA modification.

\section{Acknowledgements}

This work was supported by the National Natural Science Foundation of China (21574130, 51473161), the National Basic 
Research Program of China (973 Program-2015CB655001), and the Strategic Priority Research Program of the Chinese Academy of Sciences (Grant No. XDB12020300).

\section{References}

1 S. C. Chang, J. Liu, J. Bharathan, Y. Yang, J. Onohara and J. Kido, Multicolor organic light-emitting diodes processed by hybrid inkjet printing, Adv. Mater., 1999, 11, 734-737.

2 T. Shimoda, K. Morii, S. Seki and H. Kiguchi, Inkjet printing of light-emitting polymer displays, MRS Bull., 2003, 28, 821827.

3 Q. L. Niu, Y. X. Shao, W. Xu, L. Wang, S. H. Han, N. L. Liu, J. B. Peng, Y. Cao and J. Wang, Full color and monochrome passive-matrix polymer light-emitting diodes flat panel displays made with solution processes, Org. Electron., 2008, 9, 95-100.

4 H. Zheng, Y. N. Zheng, N. L. Liu, N. Ai, Q. Wang, S. Wu, J. H. Zhou, D. G. Hu, S. F. Yu, S. H. Han, W. Xu, C. Luo, Y. H. Meng, Z. X. Jiang, Y. W. Chen, D. Y. Li, F. Huang, J. Wang, J. B. Peng and Y. Cao, All-solution processed polymer light-emitting diode displays, Nat. Commun., 2013, 4, 1971.

5 J. Ha, J. Park, J. Ha, D. Kim, S. Chung, C. Lee and Y. Hong, Selectively modulated inkjet printing of highly conductive and transparent foldable polymer electrodes for flexible polymer light-emitting diode applications, Org. Electron., 2015, 19, 147-156.

6 R. D. Deegan, O. Bakajin, T. F. Dupont, G. Huber, S. R. Nagel and T. A. Witten, Capillary flow as the cause of ring stains from dried liquid drops, Nature, 1997, 389, 827-829.

7 Z. L. Zhang, X. Y. Zhang, Z. Q. Xin, M. M. Deng, Y. Q. Wen and Y. L. Song, Controlled inkjetting of a conductive pattern of silver nanoparticles based on the coffee-ring effect, Adv. Mater., 2013, 25, 6714-6718.

8 Z. L. Zhang and W. Y. Zhu, Controllable fabrication of a flexible transparent metallic grid conductor based on the coffee ring effect, J. Mater. Chem. C, 2014, 2, 9587-9591.

9 D. S. Eom, J. Chang, Y. W. Song, J. A. Lim, J. T. Han, H. Kim and K. Cho, Coffee-ring structure from dried graphene derivative solutions: a facile one-step fabrication route for all graphene-based transistors, J. Phys. Chem. C, 2014, 118, 27081-27090.

10 R. D. Deegan, O. Bakajin, T. F. Dupont, G. Huber, S. R. Nagel and T. A. Witten, Contact line deposits in an evaporating drop, Phys. Rev. E: Stat. Phys., Plasmas, Fluids, Relat. Interdiscip. Top., 2000, 62, 756-765.

11 R. D. Deegan, Pattern formation in drying drops, Phys. Rev. E: Stat. Phys., Plasmas, Fluids, Relat. Interdiscip. Top., 2000, 61, 475-485.

$12 \mathrm{H}$. Hu and R. G. Larson, Analysis of the effects of Marangoni stresses on the microflow in an evaporating sessile droplet, Langmuir, 2005, 21, 3972-3980.

13 D. Kim, S. Jeong, B. K. Park and J. Moon, Direct writing of silver conductive patterns: Improvement of film morphology and conductance by controlling solvent compositions, Appl. Phys. Lett., 2006, 89, 264101.
14 P. O. Babatunde, W. J. Hong, K. Nakaso and J. Fukai, Effect of solute- and solvent-derived Marangoni flows on the shape of polymer films formed from drying droplets, AIChE J., 2013, 59, 699-702.

15 B. G. de Gans and U. S. Schubert, Inkjet printing of welldefined polymer dots and arrays, Langmuir, 2004, 20, 77897793.

$16 \mathrm{H}$. Hu and R. G. Larson, Marangoni effect reverses coffeering depositions, J. Phys. Chem. B, 2006, 110, 7090-7094.

17 A. M. J. van den Berg, A. W. M. de Laat, P. J. Smith, J. Perelaera and U. S. Schubert, Geometric control of inkjet printed features using a gelating polymer, J. Mater. Chem., 2007, 17, 677-683.

18 E. L. Talbot, L. S. Yang, A. Berson and C. D. Bain, Control of the particle distribution in inkjet printing through an evaporation-driven sol-gel transition, ACS Appl. Mater. Interfaces, 2014, 6, 9572-9583.

19 D. Soltman and V. Subramanian, Inkjet-printed line morphologies and temperature control of the coffee ring effect, Langmuir, 2008, 24, 2224-2231.

20 R. B. Xing, T. L. Ye, Y. Ding, Z. C. Ding, D. G. Ma and Y. C. Han, Thickness uniformity adjustment of inkjet printed light-emitting polymer films by solvent mixture, Chin. J. Chem., 2013, 31, 1449-1454.

21 A. Chiolerio, S. Porro and S. Bocchini, Impedance hyperbolicity in inkjet-printed grapheme nanocomposites: tunable capacitors for advanced devices, Adv. Electron. Mater., 2016, 2, 1500312.

22 Y. Lin, C. F. Liu, Y. J. Song, L. Yang, W. J. Zeng, W. Y. Lai and W. Huang, Improved performances of inkjet-printed poly(3hexylthiophene) organic thin-film transistors by inserting an ionic self-assembled monolayer, RSC Adv., 2016, 6, 4097040974.

23 J. Ha, J. Park, J. Ha, D. Kim, S. Chung, C. Lee and Y. Hong, Selectively modulated inkjet printing of highly conductive and transparent foldable polymer electrodes for flexible polymer light-emitting diode applications, Org. Electron., 2015, 19, 147-156.

24 P. Wilson, C. H. Lei, C. Lekakou and J. F. Watts, Transverse charge transport in inkjet printed poly(3,4-ethylenedioxythiophene) polystyrene sulfonate (PEDOT:PSS), Org. Electron., 2014, 15, 2043-2051.

25 P. Wilson, C. Lekakou and J. F. Watts, In-plane conduction characterisation and charge transport model of DMSO codoped, inkjet printed poly(3,4-ethylenedioxythiophene): polystyrene sulfonate (PEDOT:PSS), Org. Electron., 2013, 14, 3277-3285.

26 P. Wilson, C. Lekakou and J. F. Watts, A comparative assessment of surface microstructure and electrical conductivity dependence on co-solvent addition in spin coated and inkjet printed poly(3,4-ethylenedioxythiophene): polystyrene sulphonate, Org. Electron., 2012, 13, 409-418.

27 A. D. Del Mauro, R. Diana, I. A. Grimaldi, F. Loffredo, P. Morvillo, F. Villani and C. Minarini, Polymer solar cells with inkjet-printed doped-PEDOT:PSS anode, Polym. Compos., 2013, 34, 1493-1499. 
28 T. Cheng, Y. Z. Zhang, J. P. Yi, L. Yang, J. D. Zhang, W. Y. Lai and W. Huang, Inkjet-printed flexible, transparent and aesthetic energy storage devices based on PEDOT:PSS/Ag grid electrodes, J. Mater. Chem. A, 2016, 4, 13754-13763.

29 F. Ely, C. O. Avellaneda, P. Paredez, V. C. Nogueira, T. E. A. Santos, V. P. Mammana, C. Molina, J. Brug, G. Gibson and L. Zhao, Patterning quality control of inkjet printed PEDOT:PSS films by wetting properties, Synth. Met., 2011, 161, 2129-2134.

30 A. Chiolerio, P. Rivolo, S. Porro, S. Stassi, S. Ricciardi, P. Mandracci, G. Canavese, K. Bejtka and C. F. Pirri, Inkjetprinted PEDOT:PSS electrodes on plasma-modified PDMS nanocomposites: quantifying plasma treatment hardness, RSC Adv., 2014, 4, 51477-51485.

31 J. Q. Ding, B. H. Zhang, J. H. Lu, Z. Y. Xie, L. X. Wang, X. B. Jing and F. S. Wang, Solution-processable carbazole- based conjugated dendritic hosts for power-efficient blueelectrophosphorescent devices, Adv. Mater., 2009, 21, 49834986.

32 J. Q. Ding, J. Gao, Y. X. Cheng, Z. Y. Xie, L. X. Wang, D. G. Ma, X. B. Jing and F. S. Wang, Highly efficient green-emitting phosphorescent iridium dendrimers based on carbazole dendrons, Adv. Funct. Mater., 2006, 16, 575-581.

33 S. E. Jang, C. W. Joo and J. Y. Lee, High quantum efficiency in simple blue phosphorescent organic light-emitting diodes without any electron injection layer, Thin Solid Films, 2010, 519, 906-910.

$34 \mathrm{~J}$. Li, J. C. Liu and C. J. Gao, Influence of PEDOT:PSS film doped with sorbitol on performances of organic solar cells PEDOT:PSS, Acta Phys. Sin., 2011, 60, 078803. 